\title{
Carrier Lifetime and Recombination in Long-Wavelength Quantum-Well Lasers
}

\author{
J. M. Pikal, Student Member, IEEE, C. S. Menoni, Senior Member, IEEE, H. Temkin, Fellow, IEEE, \\ P. Thiagarajan, Member, IEEE, and G. Y. Robinson, Fellow, IEEE
}

\begin{abstract}
We present a novel analysis for correcting the measured differential carrier lifetime to account for carrier population in both the barrier and separate confinement heterostructure (SCH) regions of quantum-well $(\mathrm{QW})$ lasers. This analysis uses information obtained from the measured spontaneous emission spectra to correct the measured lifetime and obtain the intrinsic well lifetime. Once the intrinsic well lifetime is obtained, the intrinsic well recombination coefficients can also be obtained. We show that the carrier population in the barrier/SCH layers can significantly affect the measured carrier lifetime and the extracted recombination coefficients. We also show that this analysis yields transparency carrier density and differential gain numbers which are very different from those obtained with the traditional analysis and much closer to what is predicted for highly strained QW lasers. These differences indicate the importance of accounting for barrier/SCH carriers on the measurement of basic QW laser material properties.
\end{abstract}

Index Terms - Charge-carrier processes, electroluminescence, optical measurement, quantum-well lasers, semiconductor lasers.

\section{INTRODUCTION}

O NE OF THE largest obstacles to reducing the cost of long-wavelength communication systems is the current need for active cooling of the semiconductor lasers used in these systems. This need arises due to the large increase in laser threshold current that is observed with an increase in device operating temperature. Therefore, a great deal of effort has been expended in trying to understand the origin of the strong temperature dependence of these lasers in hopes of reducing it. The temperature sensitivity of long-wavelength lasers has been associated with an increased contribution of Auger recombination [1], [2] and to changes in the differential gain $d g / d n$ [3], [4]. While the data supporting each mechanism as the dominant contribution to the temperature sensitivity is strong, clearly they cannot both be the dominant process. In order to study the temperature dependence of the threshold current, one must identify the recombination mechanisms that comprise the current, as well as the gain and loss properties that determine

Manuscript received October 30, 1998; revised April 28, 1999. This work was supported by the National Science Foundation under Grants ECS 9408321 and DMR 9803066.

J. M. Pikal, C. S. Menoni, and G. Y. Robinson are with the Department of Electrical and Computer Engineering, Colorado State University, Fort Collins, CO 80523 USA.

H. Temkin was with the Department of Electrical Engineering, Colorado State University, Fort Collins, CO 80523 USA. He is now with Texas Tech University, Lubbock, TX 79409 USA.

P. Thiagarajan was with the Department of Electrical Engineering, Colorado State University, Fort Collins, CO 80523 USA. He is now with Spectracom, White Bear Lake, MN 55127 USA.

Publisher Item Identifier S 1077-260X(99)06132-8. the carrier density at which one achieves lasing. While gain can be measured without direct knowledge of the carrier density, determining the material gain parameters, $d g / d n$ and $n_{0}$, the transparency carrier density, requires a method of determining carrier density in the active region. The most direct method of examining carrier recombination processes and determining carrier density is through measurement of the carrier lifetime [5].

It is, therefore, necessary to be able to measure carrier lifetime inside quantum-well (QW) active regions to fully understand the origin of the temperature sensitivity of longwavelength lasers. However, several difficulties have arisen when trying to measure the carrier lifetime in $\mathrm{QW}$ lasers. First, extracting the carrier lifetime from measurements is complicated by the additional high-frequency poles and zeros created by carrier transport across the separate confinement heterostructure $(\mathrm{SCH})$ region, and capture and escape into and out of the QW [6], [7]. These problems can be minimized by proper laser design and by using small-signal measurement techniques that reduce the frequency at which data must be acquired in order to extract the lifetime [8]. More difficult to account for is the distribution of carriers in the barrier and $\mathrm{SCH}$ regions. These carriers have a much longer lifetime due to the reduced carrier density in these regions and, therefore, affect the measured carrier lifetime and extracted recombination parameters [9]-[11].

In this paper, we develop a method for analyzing the measured carrier lifetime data based on a two-carrier-level system of rate equations for the well and barrier/SCH population instead of the traditional single-level analysis. This analysis shows that the measured lifetime is actually an effective lifetime that is a function of the well carrier lifetime, the barrier/SCH carrier lifetime, and the distribution of carriers between the two regions. We then use the measured spontaneous emission spectra to estimate the ratio of well carriers to barrier/SCH carriers, thus enabling us to obtain the intrinsic well lifetime from the measured effective lifetime. Once the well carrier lifetime is obtained, we can also obtain the intrinsic recombination parameters of the well material. Using this method of analysis, it should be possible to isolate material and structural contributions to the temperature sensitivity and, hopefully, allow for further optimization of these longwavelength devices.

\section{Rate Equations Analysis}

The standard analysis of the carrier lifetime was developed for bulk lasers and is based on a single-carrier-level rate 
equation. In order for this analysis to be valid for a QW laser, all of the carriers would have to be in the well material. While it is true that typical $\mathrm{SCH}$ transport and QW capture times are very small compared to the subthreshold carrier lifetime, carriers arriving at the $\mathrm{QW}$ region quickly come into thermal equilibrium with the carriers in the well. Therefore, in QW lasers with finite barrier height operating at nonzero temperature, there will be a fraction of carriers that occupy the three-dimensional (3-D) states above and or outside the QW regions. The ratio of the total number of carriers outside the well to that inside is dependent on the barrier height, temperature, injection level, and the ratio of the volume of the well to the volume of the barrier/SCH. One can take these 3 -D carriers into account by adding an additional carrier rate equation for the barrier and $\mathrm{SCH}$ regions. The subthreshold rate equations then become [12]

$$
\begin{aligned}
\frac{d N_{s}}{d t} & =\frac{\eta_{i} I}{q}-\frac{N_{s}}{\tau_{\text {cap }}}-\frac{N_{s}}{\tau_{s}}+\frac{N_{w}}{\tau_{\text {esc }}} \\
\frac{d N_{w}}{d t} & =\frac{N_{s}}{\tau_{\text {cap }}}-\frac{N_{w}}{\tau_{w}}-\frac{N_{w}}{\tau_{\text {esc }}} \\
\frac{d S}{d t} & =\frac{\beta N_{w}}{\tau_{w}}-\frac{S}{\tau_{p}}
\end{aligned}
$$

where $N_{w}$ is the number of carriers in the well and $\tau_{w}$ is their lifetime. $N_{s}$ and $\tau_{s}$ are the number of carriers and the lifetime of the barrier/SCH region carriers, and $\tau_{\text {cap }}$ and $\tau_{\text {esc }}$ are the effective capture and escape times into and out of the well. $S$ is the number of photons in the cavity and $\tau_{p}$ is the photon lifetime, while $\beta$ is the fraction of well carriers that recombine radiatively into the cavity mode. Finally, $\eta_{i}$ is the injection efficiency of the bias current, $I$. A small-signal analysis of these rate equations yields the theoretical frequency response of the laser

$$
\begin{aligned}
|\Delta s|=\frac{\beta \eta_{i} \Delta i}{\tau_{w}^{\prime} q V_{w}} & \frac{1}{\left|j \omega+\frac{1}{\tau_{p}}\right|} \\
& \cdot \frac{1}{\left|\left(j \omega+\frac{1}{T_{1}}\right)\left(j \omega+\frac{1}{T_{2}}\right) \tau_{\text {cap }}^{\prime}-\frac{1}{\tau_{\text {esc }}^{\prime}}\right|}
\end{aligned}
$$

with

$$
\frac{1}{T_{1}}=\frac{1}{\tau_{\text {cap }}^{\prime}}+\frac{1}{\tau_{s}^{\prime}} \quad \frac{1}{T_{2}}=\frac{1}{\tau_{\text {esc }}^{\prime}}+\frac{1}{\tau_{w}^{\prime}}
$$

where $V_{w}$ is the total well volume and the differential lifetimes $\tau^{\prime}$ are defined in the traditional way, as the derivatives of the respective recombination, capture, and escape rates with respect to the carrier density. For constant current modulation, $\Delta i$ is not a function of frequency, and all frequency dependence is explicitly shown in (2). In addition, if $\omega \ll 1 / \tau_{p}$, which is the case for these subthreshold measurements, we can then eliminate the pole due to the photon lifetime $\tau_{p}$. From the roots of the denominator, we obtain the remaining two poles. However, a numerical analysis of the two roots using typical parameter values for our $1.3-\mu \mathrm{m}$ lasers reveals that only one pole, which we call the effective differential carrier lifetime $\tau_{\text {eff }}^{\prime}$, is found to dominate over the entire frequency and bias current range of the lifetime measurement. It is this lifetime $\tau_{\text {eff }}^{\prime}$ which we obtain when we fit the measured subthreshold frequency response to a single pole response function

$$
\frac{1}{\tau_{\mathrm{eff}}^{\prime 2}}=\frac{A_{0}-\sqrt{A_{0}-4 A_{1}}}{2}
$$

where

$$
\begin{aligned}
& A_{0}=\frac{1}{T_{1}^{2}}+\frac{1}{T_{2}^{2}}+\frac{2}{\tau_{\text {cap }}^{\prime} \tau_{\text {esc }}^{\prime}} \\
& A_{1}=\frac{1}{T_{1}^{2} T_{2}^{2}}-\frac{2}{T_{1} T_{2} \tau_{\text {cap }}^{\prime} \tau_{\text {esc }}^{\prime}}+\frac{1}{\tau_{\text {cap }}^{\prime 2} \tau_{\text {esc }}^{\prime 2}} .
\end{aligned}
$$

While we find that only one pole dominates, in accordance with our measurements, the pole is not uniquely determined by the differential lifetime of the carriers in the well. The effective differential lifetime $\tau_{\text {eff }}^{\prime}$ is a function of the well differential lifetime $\tau_{w}^{\prime}$, the differential well capture $\tau_{\text {cap }}^{\prime}$, and escape times $\tau_{\text {esc }}^{\prime}$, and the recombination lifetime of the carriers in the barrier/SCH $\tau_{s}^{\prime}$. To obtain the intrinsic differential carrier lifetime of the well material, we can solve the dominant pole expression (3) for the differential well lifetime

$$
\tau_{w}^{\prime}=\frac{-S_{2}-\sqrt{S_{2}^{2}-4 S_{1} S_{3}}}{2 S_{1}}
$$

where

$$
\begin{aligned}
& S_{1}=\frac{1}{\tau_{s}^{\prime 2} \tau_{\mathrm{esc}}^{\prime 2}}-\frac{1}{T_{1}^{2} \tau_{\mathrm{eff}}^{\prime 2}}-\frac{1}{\tau_{\mathrm{esc}}^{\prime 2} \tau_{\mathrm{eff}}^{\prime 2}}-\frac{2}{\tau_{\mathrm{cap}}^{\prime} \tau_{\mathrm{esc}}^{\prime} \tau_{\mathrm{eff}}^{\prime 2}}+\frac{1}{\tau_{\mathrm{eff}}^{\prime 4}} \\
& S_{2}=\frac{2}{\tau_{\mathrm{cap}}^{\prime} \tau_{s}^{\prime} \tau_{\mathrm{esc}}^{\prime}}+\frac{2}{\tau_{s}^{\prime 2} \tau_{\mathrm{esc}}^{\prime}}-\frac{2}{\tau_{\mathrm{esc}}^{\prime} \tau_{\mathrm{eff}}^{\prime 2}} \\
& S_{3}=\frac{1}{T_{1}^{2}}-\frac{1}{\tau_{\mathrm{eff}}^{\prime 2}} .
\end{aligned}
$$

It is evident from (4) that we must know the differential capture and escape times of the carriers, as well as the differential carrier lifetime of the barrier/SCH material, to extract $\tau_{w}^{\prime}$ from the measured $\tau_{\text {eff }}^{\prime}$. Numerical analysis of (4) reveals that $\tau_{w}^{\prime}$ is only a weak function of the barrier differential lifetime. Qualitatively, this can be explained by realizing that the much lower carrier densities in the barrier/SCH region give rise to a long carrier lifetime and, therefore, little recombination. Thus, the barrier/SCH region acts more like a carrier storage region than a recombination path, and the number of carriers there is far more important than their lifetime. Thus, accurate determination of the well lifetime is possible without exact knowledge of the barrier recombination coefficients. Furthermore, we show later that the intrinsic well lifetime is mainly a function of the ratio of the differential capture to escape time and not the absolute capture and escape times. This is important, since we do not know the absolute capture and escape times, but we can obtain an estimate of the ratio of the two from the measured spontaneous emission spectra.

A steady-state solution of the carrier rate equations reveals that the ratio of the number of carriers in the barrier/SCH to that in the well $N_{s} / N_{w}$ is approximately equal to the capture to escape time ratio as given in

$$
\frac{N_{s}}{N_{w}}=\tau_{\text {cap }}\left[\frac{1}{\tau_{w}}+\frac{1}{\tau_{\mathrm{esc}}}\right] \approx \frac{\tau_{\mathrm{cap}}}{\tau_{\mathrm{esc}}} .
$$


As we will see, this approximation is valid for our lasers, since $\tau_{w}$ is larger than $\tau_{\text {esc }}$ and both decrease as the carrier density in the well increases. Since $\tau_{\text {esc }}$ becomes quite large at low bias levels and/or high barrier heights, this approximation will be less accurate, however, the correction also becomes small, since most of the carriers are in the well and not in the barrier/SCH. The ratio $\tau_{\text {cap }} / \tau_{\text {esc }}$ in (5) is the dc or steady-state ratio of capture to escape time. The ratio required to extract the intrinsic well lifetime is the ac or differential ratio. However, we can make use of previous results that showed that the ac ratio is approximately a factor of two larger than the dc ratio [13]. Therefore, if we can find the ratio of the total number of carriers in the barrier/SCH to that in the well, we can extract the differential lifetime of the carriers in the well.

The analysis just described requires the ratio of barrier/SCH carriers to well carriers. This ratio is a strong function of laser bias and temperature and, therefore, must be obtained under the same conditions as the lifetime measurement. We show next that the ratio of well carriers to barrier/SCH carriers can be determined from the measured spontaneous emission spectra. The integrated spontaneous emission intensity is proportional to the density of carriers, and the emission spectra provides information on how the carriers are distributed in energy. If we assume the traditional carrier density squared dependence of the integrated spontaneous emission intensity, we can write the ratio of the barrier/SCH spontaneous emission to the well emission as

$$
R_{t}=\frac{V_{s} B_{s} n_{s}^{2}}{V_{w} B_{w} n_{w}^{2}}=\frac{V_{w} B_{s} N_{s}^{2}}{V_{s} B_{w} N_{w}^{2}}
$$

where $V_{s}$ is the total volume of the barrier and $\mathrm{SCH}$ regions, and $B_{s}$ and $B_{w}$ are the quadratic (radiative) recombination coefficients of the barrier/SCH and the well, respectively. The carrier densities in the barrier/SCH and well are $n_{s}$ and $n_{w}$, respectively. Rearranging (6) and combining it with (5) yields

$$
\frac{\tau_{\text {cap }}}{\tau_{\text {esc }}}=\frac{N_{s}}{N_{w}}=\sqrt{R_{t} \frac{V_{s} B_{w}}{V_{w} B_{s}}} .
$$

Thus, by measuring the ratio of barrier/SCH spontaneous emission to well spontaneous emission $R_{t}$ and knowing $B_{w} / B_{s}$, we can obtain the ratio of capture to escape times $\tau_{\text {cap }} / \tau_{\text {esc }}$. While $B_{s}$ is known to be approximately $1.0 \times 10^{-10} \mathrm{~cm}^{3} / \mathrm{s}$ [14], $B_{w}$ is one of the parameters we are trying to determine. Therefore, we must perform our analysis in a self-consistent manner by choosing an initial value for $B_{w}$, finding the intrinsic recombination parameters, and then feeding the new $B_{w}$ back into (7) until we converge on a solution. While this analysis is somewhat complicated, we always converged to the same solution in just a few iterations, regardless of the initial choice of recombination parameters.

\section{MEASUREMENTS AND RESUlTS}

Differential carrier lifetime, spontaneous emission, and gain measurements were performed on $1.3-\mu \mathrm{m}$ InAsP-InGaAsP MQW SCH buried heterostructure $(\mathrm{BH})$ lasers with cleaved facets. These lasers were grown by gas-source molecular beam epitaxy (GSMBE) with the structure shown in Fig. 1 and

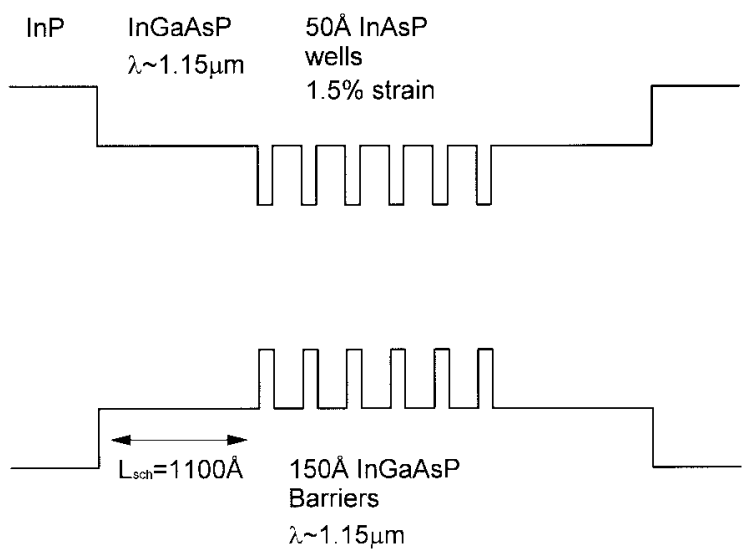

Fig. 1. Schematic diagram of the structure of the 1.3- $\mu \mathrm{m}$ InAsP-InGaAsP MQW SCH lasers used in this study.

described in more detail elsewhere [15]. The lasers were buried with metal-organic chemical vapor deposition (MOCVD)grown InP, cleaved and mounted on a gold-plated copper heat sink for temperature control. These structures have yielded devices with threshold currents as low as $1.1 \mathrm{~mA}$ at $20^{\circ} \mathrm{C}$ and $6.1 \mathrm{~mA}$ at $100^{\circ} \mathrm{C}$ on $125-\mu \mathrm{m}$-long lasers with highly reflective (HR) coating on both facets [16]. Threshold currents for the $500-\mu \mathrm{m}$-long cleaved facet lasers used in this study were around $8.1 \mathrm{~mA}$ at $20^{\circ} \mathrm{C}$. Note that the laser structure used has a larger than optimal barrier/SCH bandgap wavelength of 1.15 $\mu \mathrm{m}$. This translates into a smaller confinement energy and, thus, more barrier/SCH carriers than typical $1.3-\mu \mathrm{m}$ lasers. The result of additional barrier/SCH carriers is a somewhat larger difference between the measured effective parameters of the single rate equation analysis and the intrinsic material parameters extracted from the two-level rate equation analysis.

\section{A. Spontaneous Emission}

The spontaneous emission was collected from the side of the laser through a pinhole in order to remove any light scattered from the facets. The collected light was chopped and focused onto the entrance slit of a single pass grating spectrometer equipped with an $\mathrm{LN}_{2}$-cooled $\mathrm{InGaAs}$ detector and lock-in for signal detection. The solid lines in Fig. 2 show typical measured spontaneous emission spectra obtained from our InAsP MQW lasers at low bias [Fig. 2(a)] and near threshold [Fig. 2(b)]. Due to the large overlap of the well heavy hole, light hole, and barrier emission, one cannot simply numerically integrate the well and barrier/SCH intensity separately. Therefore, we fit the measured spontaneous emission spectra to a spectra calculated by summing over all possible transitions and assuming that the $k$-selection rule holds. For a $\mathrm{QW}$, the spontaneous emission spectra is of the form [14]

$$
\begin{array}{r}
R_{\mathrm{sp}}^{2 D}(E)=\frac{16 \pi^{2} \eta_{r} q^{2} m_{r}\left|M_{b}\right|^{2} P}{m_{0}^{2} \epsilon_{0} h^{4} c^{3} L_{w}} f_{c}\left(\frac{m_{r}}{m_{e}}\left(E-E_{1}\right)\right) \\
\cdot f_{v}\left(\frac{m_{r}}{m_{h}}\left(E-E_{1}\right)\right) E \Phi\left(E-E_{1}\right)
\end{array}
$$


(a)

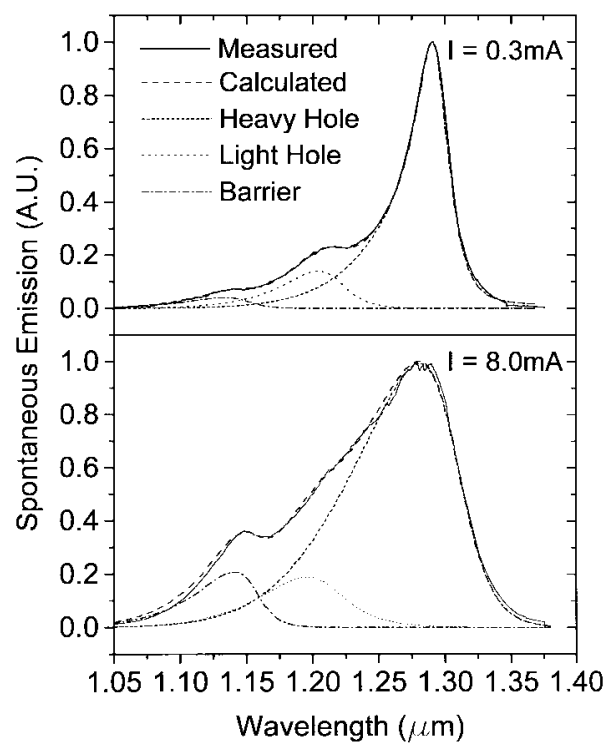

Fig. 2. Measured and calculated spontaneous emission spectra showing heavy hole, light hole, and barrier contributions for (a) low bias, $I=0.3$ $\mathrm{mA}$, and (b) high bias, $I=8.0 \mathrm{~mA}$.

where

$$
\left|M_{b}\right|^{2}=\frac{m_{0}^{2} E_{1}\left(E_{1}+\Delta\right)}{12 m_{c}\left(E_{1}+\frac{2}{3} \Delta\right)} .
$$

In this equation, $\Phi$ is the Heaviside step function, and $E_{1}$ is the energy separation of the transition of interest, for instance, the first confined electron level to the first heavy or light hole level. The term $P$ is the polarization-dependent part of the matrix element, which we assume to be energy independent and equal to $2 / 3$ and $1 / 3$, for the heavy hole and light hole transitions, respectively. The meanings of all other parameters are those given in [14]. A similar equation describes the bulklike barrier/SCH states. Adding this bulk term to the QW heavy and light hole bands and then broadening the spectra using a hyperbolic sine line-shape function [17] yields the calculated spontaneous emission spectra. The spontaneous emission at different bias levels can be calculated by changing the Fermi levels and broadening parameters in the calculation. Clearly, there are many model parameters in this calculation, some of which are not known with great accuracy. Fortunately, we only need the appropriate shape of each band in order to find the ratio of integrated barrier/SCH to well emission.

Fig. 2 shows the calculated heavy hole, light hole, and barrier spontaneous emission as well as the sum of these emission bands for comparison to the measured data. Good agreement is achieved at the two bias extremes shown for illustration, as well as for all bias levels in between. In the case of low bias [Fig. 2(a)], the measured and calculated curves are almost indistinguishable from each other, while near threshold [Fig. 2(b)], the calculated spontaneous emission has slightly increased emission from the high energy tail of the barrier compared to the measured spectra. We have not been able to explain or correct for this effect, but it is small and should not add significant error into the determination of $R_{t}$. One can easily see from Fig. 2 that the amount of emission from the barrier increases significantly with increasing bias current. The

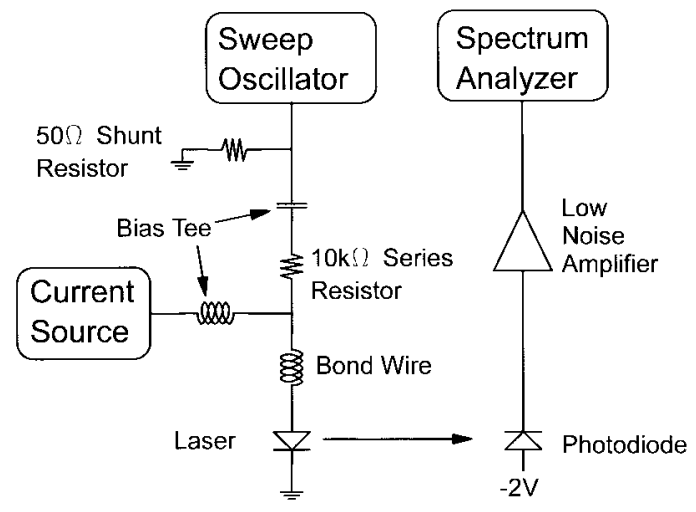

Fig. 3. Diagram of the impedance-independent optical carrier lifetime measurement setup showing the impedance stabilization circuit and bias arrangement.

ratio of integrated barrier/SCH to well emission $R_{t}$ varies from about $6.5 \%$ at low bias to nearly $17 \%$ just below threshold for the $1.3-\mu \mathrm{m}$ InAsP lasers investigated.

\section{B. Carrier Lifetime}

The differential carrier lifetime measurements where carried out using a modified optical technique that allows us to perform the measurements with nearly constant current modulation. This removes the recently reported error in the measured differential carrier lifetime that occurs at low bias levels [18], [19]. This error is due to a changing modulation current caused by the large and frequency-dependent laser impedance at low bias. In our technique, we provide a constant modulation current to the laser by adding an impedance stabilization circuit prior to the laser diode, as shown in Fig. 3. The circuit consists of a $10-\mathrm{k} \Omega$ series resistance, which is much larger than the frequency-dependent impedance of the laser, allowing a nearly constant current into the diode. In addition, a $50-\Omega$ shunt resistor is placed before the series resistor to provide a matched load for the source and to reduce the crosstalk of the system. The receiver, cable, and source nonlinearity are calibrated out by subtracting off the measured above threshold frequency response from the subthreshold response curves. This impedance-independent optical technique, which is described in more detail elsewhere [8], provides very clean calibrated modulation response curves, as shown in Fig. 4. As we can see from Fig. 4, it is only necessary to measure the laser response to $200 \mathrm{MHz}$ in order to obtain a reliable fit to the theoretical single-pole response. Capture and escape into and out of the QW and carrier transport across the SCH region can create additional high-frequency poles and zeros in the response. Being able to obtain the lifetime from data collected at such low frequencies is a distinct advantage of our technique for applications involving QW lasers.

The differential carrier lifetime was measured on our InAsP lasers at bias levels from 0.2 to $8.0 \mathrm{~mA}\left(I_{\mathrm{th}}=8.1 \mathrm{~mA}\right)$ using the procedure described above. If one uses the traditional single-carrier-level rate equation analysis, the recombination parameters $A_{\text {eff }}, B_{\text {eff }}$, and $C_{\text {eff }}$, are obtained from the simultaneous fit of the measured differential lifetime $\tau_{\text {eff }}^{\prime}$ to the current versus carrier density (9a) and lifetime as a function of carrier 


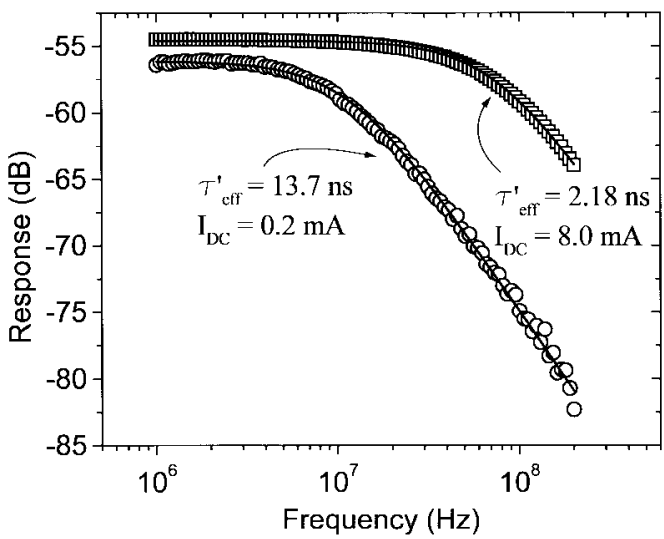

Fig. 4. Typical subthreshold modulation responses of 1.3- $\mu \mathrm{m}$ InAsP MQW laser shown with corresponding single-pole fit and extracted effective lifetime.

(a)

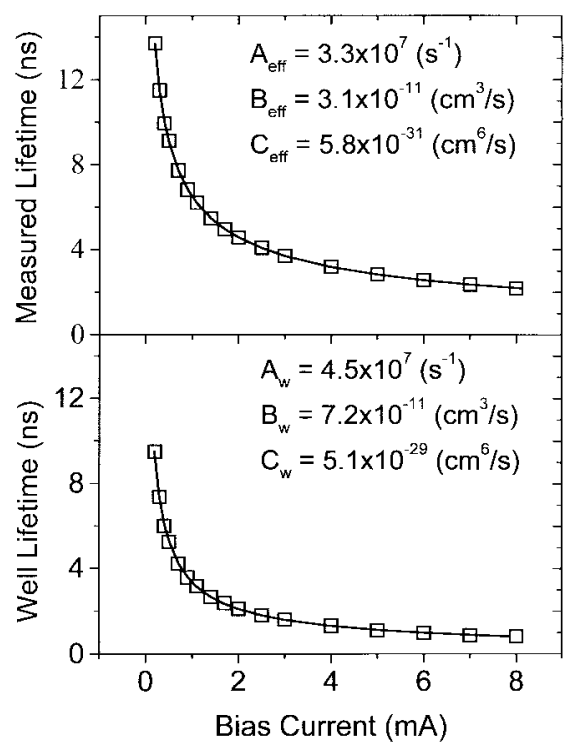

Fig. 5. (a) Effective differential carrier lifetime versus bias current with single-rate equation fit and extracted effective recombination parameters. (b) Intrinsic well differential carrier lifetime versus bias current with two-carrier-level rate equation fit and extracted intrinsic well recombination parameters.

density (9b)

$$
\begin{aligned}
I & =\frac{q V_{w}}{\eta_{i}}\left(A_{\mathrm{eff}} n_{\mathrm{eff}}+B_{\mathrm{eff}} n_{\mathrm{eff}}^{2}+C_{\mathrm{eff}} n_{\mathrm{eff}}^{3}\right) \\
\frac{1}{\tau_{\mathrm{eff}}^{\prime}} & =A_{\mathrm{eff}}+2 B_{\mathrm{eff}} n_{\mathrm{eff}}+3 C_{\mathrm{eff}} n_{\mathrm{eff}}^{2} .
\end{aligned}
$$

Here, we have explicitly written the recombination parameters, as well as the differential carrier lifetime and the carrier density, as effective parameters. Using this procedure, we obtain the fit shown by the solid line in Fig. 5(a) along with the effective recombination parameters. Although the fit is good, the extracted recombination parameters are not useful, since they are not material parameters, but include the effect of the barrier/SCH carrier population and, therefore, the laser structure.

Using the two-carrier-level rate equation analysis described in Section II, we can rewrite (9a) and (9b) to take into account the carrier distribution in the barrier/SCH layers. The current and lifetime equations then become

$$
\begin{aligned}
I= & \frac{q}{\eta_{i}}\left[V_{w}\left(A_{w} n_{w}+B_{w} n_{w}^{2}+C_{w} n_{w}^{3}\right)\right. \\
& \left.+V_{s}\left(A_{s} n_{s}+B_{s} n_{s}^{2}+C_{s} n_{s}^{3}\right)\right] \\
\frac{1}{\tau_{w}^{\prime}}= & A_{w}+2 B_{w} n_{w}+3 C_{w} n_{w}^{2} .
\end{aligned}
$$

In order to limit the number of free parameters in this fit, we assume that the nonradiative Shockley-Hall-Read recombination coefficients $A_{w}$ and $A_{s}$ are more dependent on interface quality than material quality. As such, they are inseparable, and we set them equal and obtain their value from the fit. The radiative coefficient for the barrier $B_{s}$ is set to $1 \times 10^{-10}$ $\mathrm{cm}^{3} / \mathrm{s}$ as before. The Auger coefficient of the barrier/SCH $C_{s}$ is not well known, but we know it should be less than the Auger coefficient of $1.3-\mu \mathrm{m}$ bulk [19] material, since the bandgap is larger. We therefore choose a value of $1 \times 10^{-29}$ $\mathrm{cm}^{6} / \mathrm{s}$ for $C_{s}$, knowing it will have little effect on the results due to the low carrier densities in the barrier/SCH layers. The differential carrier lifetime in the well $\tau_{w}^{\prime}$ is obtained, in an iterative fashion, from the measured effective lifetime $\tau_{\text {eff }}^{\prime}$, the measured ratio of barrier/SCH emission $R_{t}$, and (4)-(7).

The intrinsic well differential carrier lifetime versus bias current is shown in Fig. 5(b). By comparing the curves in Fig. 5, we see that the effective lifetime is longer than the well lifetime by approximately $40 \%$ at low bias and by a factor of two at high bias. The fact that the effective lifetime is longer than the intrinsic well lifetime in not surprising, since the effective lifetime is a combination of the well lifetime and the longer, due to diluted carrier density, barrier/SCH lifetime. In addition, we expect the difference between the effective lifetime and the well lifetime to increase at high bias, since the fraction of carrier in the barrier/SCH increases. However, the most important result is the considerable difference in the extracted recombination parameters. The $A$ coefficient is the least affected, as it increases by about $30 \%$ to $4.5 \times 10^{7}$ $\mathrm{s}^{-1}$. This value is still fairly low and indicates good quality barrier-well interfaces, as well as good material quality, in both the well and barrier/SCH layers. The $B$ coefficient increases roughly $60 \%$ to a more reasonable $0.72 \times 10^{-10}$ $\mathrm{cm}^{3} / \mathrm{s}$, while the $C$ coefficient increases almost two orders of magnitude to $5.1 \times 10^{-29} \mathrm{~cm}^{6} / \mathrm{s}$. Again, the trends in the coefficients are as expected, since the largest changes are in the coefficients most sensitive to carrier density, i.e., $C$.

While our lasers are probably worst case due to their low barrier height, as discussed previously, the results show that considerable underestimation of the $C$ coefficient can result if the barrier/SCH population is not taken into account. This is particularly true at higher temperatures, even in lasers with larger barrier/SCH bandgap energy, and may contribute to the unexpectedly low temperature dependence of the $C$ coefficient that has been measured [4], [20].

We are now in a position to investigate some of the assumptions made earlier in the analysis and determine the sensitivity of our method to some of the parameters that we had to estimate. In Section II, we stated that the differential well lifetime was mainly a function of the ratio of the capture to escape time and not very sensitive to the absolute values 
TABLE I

Extracted Well Recombination Parameters versus Capture Time

\begin{tabular}{c||c|c|c|c}
\hline$\tau_{\text {cap }}$ & $0.1 \mathrm{ps}$ & $1.0 \mathrm{ps}$ & $10 \mathrm{ps}$ & $100 \mathrm{ps}$ \\
\hline \hline $\mathrm{A}_{\mathrm{w}} \times 10^{-7}$ & 4.47 & 4.47 & 4.49 & 4.70 \\
\hline $\mathrm{B}_{\mathrm{w}} \times 10^{10}$ & 0.719 & 0.719 & 0.709 & 0.623 \\
\hline $\mathrm{C}_{\mathrm{w}} \times 10^{29}$ & 5.05 & 5.06 & 5.02 & 4.76 \\
\hline
\end{tabular}

TABLE II

Extracted Well ReCOMBINATION PARAmeters VERSUS BARRIER/SCH COEFFICIENTS

\begin{tabular}{l||l|l|l||l|l|l}
\hline & \multicolumn{5}{|c||}{$\mathrm{C}_{\mathrm{s}} \times 10^{29}$} & \multicolumn{4}{|c}{$\mathrm{B}_{\mathrm{s}} \times 10^{10}$} \\
& 0.2 & 1.0 & 5.0 & 0.8 & 1.0 & 1.2 \\
\hline \hline $\mathrm{A}_{\mathrm{w}} \times 10^{-7}$ & 4.49 & 4.47 & 4.36 & 4.67 & 4.47 & 4.31 \\
\hline $\mathrm{B}_{\mathrm{w}} \times 10^{10}$ & 0.709 & 0.719 & 0.775 & 0.737 & 0.719 & 0.698 \\
\hline $\mathrm{C}_{\mathrm{w}} \times 10^{29}$ & 5.03 & 5.06 & 5.23 & 6.80 & 5.06 & 3.95 \\
\hline
\end{tabular}

of these times. Table I shows the intrinsic recombination parameters obtained from the analysis using capture times ranging from 0.1 to $100 \mathrm{ps}$. In each case, the escape times were allowed to vary with bias according to the measured bias dependence of $R_{t}$ in (7). Table I shows that, even with capture times covering three orders of magnitude, the change in the intrinsic recombination coefficients is less than $15 \%$, illustrating the insensitivity of our technique to the absolute value of the capture and escape times.

We also investigated the sensitivity of our analysis to the barrier/SCH recombination parameters fixed in Section III-B to reduce the number of fitting parameters. Table II shows the intrinsic recombination parameters obtained from the analysis using various values for the barrier/SCH recombination coefficients $B_{s}$ and $C_{s}$. Note that, as described previously, $A_{s}$ is not varied, since its value was not fixed in (10b), but was determined from the fitting procedure. From Table II, we see that varying $C_{s}$ from $2.0 \times 10^{-30} \mathrm{~cm}^{6} / \mathrm{s}$ to $5.0 \times 10^{-29}$ $\mathrm{cm}^{6} / \mathrm{s}$ resulted in no more than $9 \%$ variation in the extracted intrinsic recombination parameters.

The parameter to which our method is most sensitive is $B_{s}$. This is not surprising, since $B_{s}$ not only contributes to the current in (10a), but is also important in determining the capture to escape ratio in (7) and, thus, the ratio of barrier/SCH carriers to well carriers. Fortunately, the bulk radiative recombination coefficient is also a parameter that varies little with material composition and quality and, therefore, we know it more accurately than $C_{s}$ or $\tau_{\text {cap. }}$. The typical number for $B_{s}$ in InGaAsP alloys is $1.0 \times 10^{-10} \mathrm{~cm}^{3} / \mathrm{s}$ and we therefore vary $B_{s}$ from $0.8 \times 10^{-10} \mathrm{~cm}^{3} / \mathrm{s}$ to $1.2 \times 10^{-10} \mathrm{~cm}^{3} / \mathrm{s}$. From Table II, it is clear that this resulted in little change for the fitted $A_{w}(\sim 8 \%)$ and $B_{w}(\sim 5 \%)$ coefficients. The $C_{w}$ coefficient, however, varied from $3.95 \times 10^{-29} \mathrm{~cm}^{6} / \mathrm{s}$ to $6.80 \times 10^{-29}$ $\mathrm{cm}^{6} / \mathrm{s}$, an increase of about $70 \%$. While this is a fairly large range, it is quite small compared to the approximately two-

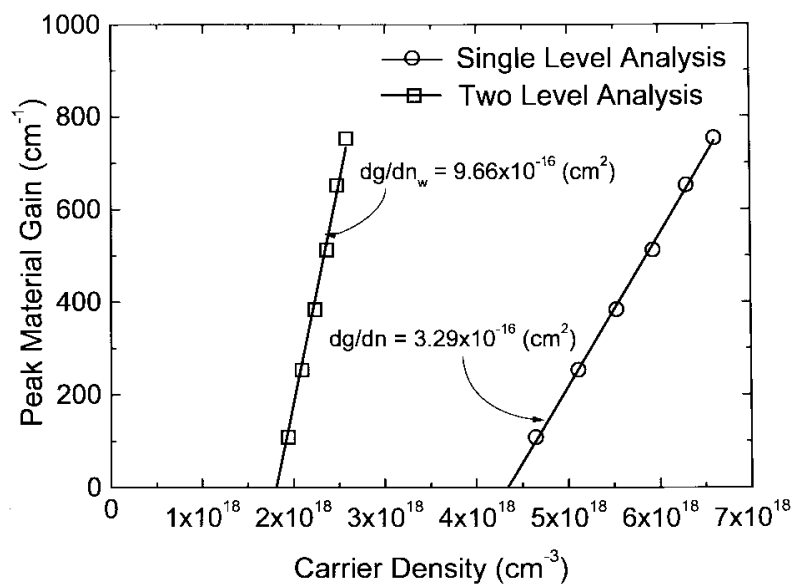

Fig. 6. Peak material gain versus carrier density calculated from both the single- and two-carrier-level-rate equation analysis techniques.

orders-of-magnitude difference in $C_{w}$ obtained between the single-level analysis and the two-level rate equation analysis. It is clear from this procedure that our analysis is reasonably insensitive to the parameters which we have had to estimate.

\section{Gain}

As a final example of the effect that neglecting the barrier/SCH carrier distribution can have on laser material evaluation, we measured the material gain of the $1.3-\mu \mathrm{m}$ InAsP lasers and extracted the transparency carrier density $n_{O}$ and the differential gain $d g / d n$. The amplified spontaneous emission (ASE) spectra were measured in a manner similar to the spontaneous emission spectra, with the exception that the light was collected from the facet for the ASE measurement. The gain was then determined from the peak-to-valley ratio of the ASE using the technique of Hakki and Paoli [21]. A plot of the measured peak material gain versus carrier density is shown in Fig. 6. We have plotted the gain versus the carrier density obtained from the simple, single-level rate equation analysis and the two-level rate equation analysis. The deduced transparency carrier density is found to drop from a rather large $4.3 \times 10^{18}$ $\mathrm{cm}^{-3}$ in the former to a more reasonable $1.8 \times 10^{18} \mathrm{~cm}^{-3}$ in the latter. The large number obtained in the single-level rate equation analysis is a reflection of the large carrier population in the barrier/SCH that contributes only to carrier population and not to population inversion for the well material. In addition, the differential gain increases from a bulk-like value of $3.3 \times 10^{-16} \mathrm{~cm}^{2}$ to $9.7 \times 10^{-16} \mathrm{~cm}^{2}$. The larger intrinsic differential gain number is much closer to that expected from a highly strained QW laser material and is indicative of what could be achieved with larger bandgap barrier/SCH layers.

\section{CONCLUSIONS}

We have presented a novel analysis for correcting the measured differential carrier lifetime to account for carrier population in both the barrier and $\mathrm{SCH}$ regions of QW lasers. We used two carrier levels in the rate equation analysis and the measured spontaneous emission spectra to extract the intrinsic well lifetime and, therefore, the intrinsic recombination parameters of the well. We showed that this new analysis yields significantly different well lifetimes, well recombination 
coefficients, as well as transparency carrier densities and differential gains, compared to values obtained from a single rate equation analysis. Our analysis showed an increase of nearly two orders of magnitude in the Auger coefficient $C$ over the standard, single-level rate equation analysis and a factor of three increase in the well differential gain. These changes may have a significant impact on the analysis of the temperature sensitivity of long-wavelength lasers and indicate the importance of the barrier/SCH carriers on the measurement of basic laser material properties.

\section{REFERENCES}

[1] W. Fang, M. Hattendorf, S. L. Chuang, J. Minch, C. S. Chang, C. G. Bethea, and Y. K. Chen, "Analysis of temperature sensitivity in semiconductor lasers using gain and spontaneous emission measurements," Appl. Phys. Lett., vol. 70, no. 7, pp. 796-798, 1997.

[2] S. J. Sweeney, A. F. Phillips, A. R. Adams, E. P. O'Reilly, and P. J. A. Thijs, "The effect of temperature dependent processes on the performance of $1.5 \mu \mathrm{m}$ compressively strained InGaAs(P) MQW semiconductor diode lasers," IEEE Photon. Technol. Lett., vol. 10, pp. 1076-1078, Aug. 1998

[3] D. A. Ackerman, P. A. Morton, G. E. Shtengel, M. S. Hybertsen, R. F. Kazarinov, T. Tanbun-Ek, and R. A. Logan, "Analysis of $T_{0}$ in $1.3 \mu \mathrm{m}$ multi-quantum-well and bulk active lasers," Appl. Phys. Lett., vol. 66, no. 20, pp. 2613-2615, 1995.

[4] Y. Zou, J. S. Osinski, P. Grodzinski, P. D. Dapkus, W. C. Rideout, W. F. Sharfin, J. Schlafer, and F. D. Crawford, "Experimental study of auger recombination, gain, and temperature sensitivity of $1.5 \mu \mathrm{m}$ compressively strained semiconductor lasers," IEEE J. Quantum Electron., vol. 29, pp. 1565-1575, June 1993.

[5] R. Olshansky, C. B. Su, J. Manning, and W. Powazinik, "Measurement of raditive and nonradiative recombination rates in InGaAsP and AlGaAs light sources," IEEE J. Quantum Electron., vol. QE-20, pp. 838-854, Aug. 1984.

[6] S. Weisser, I. Esquivias, P. J. Tasker, J. D. Ralston, B. Romero, and J. Rosenzweig, "Impedance characteristics of quantum-well lasers," IEEE Photon. Technol. Lett., vol. 6, pp. 1421-1423, Dec. 1994.

[7] I. Esquivias, S. Weisser, B. Romero, J. D. Ralston, and J. Rosenzweig, "Carrier capture and escape times in $\mathrm{In}_{0.35} \mathrm{Ga}_{0.65} \mathrm{As}-\mathrm{GaAs}$ multiquantum-well lasers determined from high-frequency electrical impedance measurements," IEEE Photon. Technol. Lett., vol. 8, pp. 1294-1296, Oct. 1996.

[8] J. M. Pikal, C. S. Menoni, H. Temkin, P. Thiagarajan, and G. Y. Robinson, "Impedance independent optical carrier lifetime measurements in semiconductor lasers," Rev. Sci. Instrum., vol. 69, no. 12, pp. 4247-4248, Dec. 1998.

[9] A. P. Ongstad, D. J. Gallant, and G. C. Dente, "Carrier lifetime saturation in InGaAs single quantum wells," Appl. Phys. Lett., vol. 66, no. 20, pp. 2730-2732, 1995.

[10] Y. C. Chen, P. Wang, J. J. Coleman, D. Bour, K. K. Lee, and R. G. Waters, "Carrier recombination rates in strained-layer InGaAs-GaAs quantum wells," IEEE J. Quantum Electron., vol. 27, pp. 1451-1454, June 1991.

[11] T. Yamamoto, T. Odagawa, K. Tanaka, and S. Ogita, "Influence of separate confinement heterostructures on the effective carrier recombination coefficient in quantum well laser structures," Appl. Phys. Lett., vol. 71, no. 11, pp. 1543-1545, 1997.

[12] L. A. Coldren and S. W. Corzine, Diode Lasers and Photonic Integrated Circuits. New York: Wiley, 1995.

[13] C.-Y. Tsai, C.-Y. Tsai, Y.-H. Lo, and L. F. Eastman, "Carrier DC and AC capture and escape times in quantum-well lasers," IEEE Photon. Technol. Lett., vol. 7, pp. 599-601, June 1995.

[14] G. P. Agrawal and N. K. Dutta, Semiconductor Lasers, 2nd ed. New York: Van Nostrand Reinhold, 1993.

[15] P. Thiagarajan, A. A. Bernussi, H. Temkin, G. Y. Robinson, A. M. Sergent, and R. Logan, "Growth of $1.3 \mu \mathrm{m}$ InAsP/InGaAsP laser structures by gas source molecular beam epitaxy," Appl. Phys. Lett., vol. 67, no. 25, pp. 3676-3678, 1995.

[16] P. Thiagarajan, G. E. Giudice, H. Temkin, and G. Y. Robinson, "Low threshold current $1.3 \mu \mathrm{m}$ InAsP/InGaAsP lasers grown by gas-source molecular beam epitaxy," Electron. Lett., vol. 32, no. 12, pp. 1103-1105, 1996.

[17] W. W. Chow, S. W. Koch, and M. Sargent III, Semiconductor-Laser Physics. Berlin, Germany: Springer-Verlag, 1994.
[18] G. E. Shtengel, D. A. Ackerman, and P. A. Morton, "True carrier lifetime measurements of semiconductor lasers," Electron. Lett., vol. 31, no. 20 , pp. 1747-1748, 1995.

[19] G. E. Shtengel, D. A. Ackerman, P. A. Morton, E. J. Flynn, and M. S. Hybertsen, "Impedance-corrected carrier lifetime measurements in semiconductor lasers," Appl. Phys. Lett., vol. 67, no. 11, pp. 1506-1508, 1995.

[20] G. Fuchs, C. Schiedel, A. Hangleiter, V. Harle, and F. Scholz, "Auger recombination in strained and unstrained InGaAs/InGaAsP multiple quantum-well lasers," Appl. Phys. Lett., vol. 62, no. 4, pp. 396-398, 1993.

[21] B. W. Hakki and T. L. Paoli, "Gain spectra in GaAs doubleheterostructure injection lasers," J. Appl. Phys., vol. 46, no. 3, pp. 1299-1306, 1975.

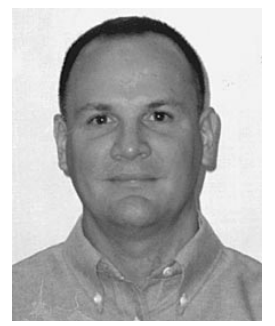

J. M. Pikal (S'95) received the B.S. degree in electrical engineering from Purdue University, West Lafayette, IN, in 1988 and the M.S. degree from the University of Colorado, Boulder, in 1993. He is currently working towards the Ph.D. degree at Colorado State University, Fort Collins, studying the temperature dependence of carrier lifetime, recombination, and gain in long-wavelength semiconductor lasers.

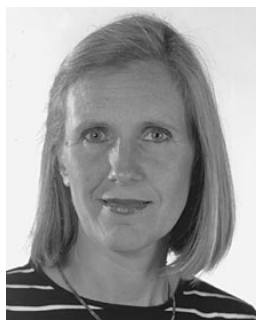

C. S. Menoni (M'93-SM'98) received the Ph.D degree in physics from Colorado State University, Fort Collins, in 1987.

In 1991, she joined the Department of Electrical and Computer Engineering, Colorado State University, where she is currently an Associate Professor. Her research interests are in the characterization of semiconductor lasers and related heterostructures materials, with emphasis on the study of carrier recombination mechanisms and carrier transport in long-wavelength lasers. Her current research also encompasses the study of carrier localization and recombination in widebandgap InGaN/GaN and II-VI heterostructures, as well as the investigation of the electronic structure of InP quantum dots. She has authored or coauthored numerous technical papers, two book chapters, and several conference presentations.

Prof. Menoni is a member the American Physical Society and the Optical Society of America.

H. Temkin (F'93), photograph and biography unavailable at the time of publication.

P. Thiagarajan (M'98) received the Ph.D. degree in electrical and computer engineering from Colorado State University, Fort Collins, in 1996.

He is currently a Member of the Technical Staff at Spectracom, St. Paul, MN. His areas of interest are the epitaxy of III-V compounds, semiconductor lasers, optics, and high-speed characterization of materials and devices.

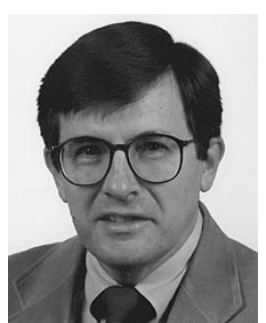

G. Y. Robinson (S'66-M'69-SM'93-F'96) received the B.E.S. degree from the University of Texas, Austin, in 1965 and the M.S. and Ph.D. degrees from the University of California, Berkeley, in 1967 and 1969, respectively.

From 1970 to 1984, he was an Assistant Professor, Associate Professor, and Professor of Electrical Engineering at the University of Minnesota, Minneapolis, where he conducted research on electrical and metallurgical characterization of metal-semiconductor contacts on $\mathrm{Si}$ and the III-V semiconductors. In 1984, he started a new research group at Colorado State University, Fort Collins, where he is currently a Professor of Electrical and Computer Engineering and is involved in the growth of III-V heterostructures by gas-source molecular beam epitaxy. He has taught short courses in the U.S. and Europe and has served on organizing committees for numerous conferences on the III-V semiconductors.

Prof. Robinson is a member of the American Vacuum Society. 